\title{
Asymptotic formulas for class number sums of indefinite binary quadratic forms in arithmetic progressions
}

\author{
Yasufumi Hashimoto *
}

\begin{abstract}
It is known that there is a one-to-one correspondence between equivalence classes of primitive indefinite binary quadratic forms and primitive hyperbolic conjugacy classes of the modular group. Due to such a correspondence, Sarnak obtained the asymptotic formula for the class number sum in order of the fundamental unit by using the prime geodesic theorem for the modular group. In the present paper, we propose asymptotic formulas of the class number sums over discriminants in arithmetic progressions. Since there are relations between the arithmetic properties of the discriminants and the conjugacy classes in the finite groups given by the modular group and its congruence subgroups, we can get the desired asymptotic formulas by arranging the Tchebotarevtype prime geodesic theorem. While such asymptotic formulas were already given by Raulf, the approaches are quite different, the expressions of the leading terms of our asymptotic formulas are simpler and the estimates of the reminder terms are sharper.
\end{abstract}

\section{Introduction}

For an integer $D$, let $h(D)$ be the class number of $D$ in the narrow sense. In Sects. 302-304 of [G], Gauss stated the mean value formula for $h(D)$ without proof. His formula for $D<0$ was proven by Lipschitz [Li] and Mertens [Me] and was improved by Vinogradov [Vi]. For $D>0$, Siegel [Si] proved that

$$
\sum_{0<D<x} h(D) \log \epsilon(D) \sim \frac{\pi^{2}}{18 \zeta(3)} x^{3 / 2} \quad \text { as } \quad x \rightarrow \infty,
$$

where $\epsilon(D)$ is the fundamental unit of $D$ in the narrow sense and $\zeta(3):=\sum_{n \geq 1} n^{-3}$, and Shintani Sh] improved it. Such an asymptotic formulas has been further improved and extended in several ways by the theory of prehomogeneous vector spaces (see [Sh], [Da], [GH] etc).

On the other hand, Sarnak [Sa1] obtained the following asymptotic formula.

$$
\sum_{D>0, \epsilon(D)<x} h(D) \log \epsilon(D) \sim \frac{1}{2} x^{2} \quad \text { as } \quad x \rightarrow \infty .
$$

*Partially support by JSPS Grant-in-Aid for Young Scientists (B) no. 20740027.

MSC2000: primary: 11E41; secondary: 11M36 
This yields that

$$
\sum_{D>0, \epsilon(D)<x} h(D) \sim \operatorname{li}\left(x^{2}\right) \quad \text { as } \quad x \rightarrow \infty
$$

where $\operatorname{li}(x):=\int_{2}^{x}(\log t)^{-1} d t$. The asymptotic formulas (1.2) and (1.3) follows from the prime geodesic theorem (see, e.g. [Se] and $[\mathrm{He}]$ ) for $\mathrm{SL}_{2}(\mathbb{Z})$;

$\#\left\{[\gamma]\right.$ : the primitive hyperbolic conjugacy classes of $\mathrm{SL}_{2}(\mathbb{Z})$,

the larger eigenvalue of $\gamma$ is less than $x\} \sim \operatorname{li}\left(x^{2}\right)$ as $x \rightarrow \infty$

and the one-to-one correspondence between the equivalence classes of the primitive indefinite binary quadratic forms and the primitive conjugacy classes of $\mathrm{SL}_{2}(\mathbb{Z})$. Such asymptotic formulas have been extended to the binary quadratic forms over imaginary quadratic fields [Sa2] and the ternary quadratic forms [DH] by the theory of trace formulas.

In the present paper, we study the growth of Sarnak-type class number sums over $D \equiv$ $a$ mod $n$ for given $a$ and $n$. The approach is as follows; (i) describe the relation between the arithmetic property of the discriminants and the conjugacy classes in PSL2( $\mathbb{Z} / \mathrm{n} \mathbb{Z}$ ), (ii) write down the Tchebotarev-type prime geodesic theorems ([Sa1] and [Su]) as asymptotic formulas for partial class number sums and (iii) arrange such asymptotic formulas. The main result, Theorem 4.8, gives the detail expressions of the coefficients of the leading terms and (not necessarily best-possible but) non-trivial estimates of the reminder terms.

For such asymptotic formulas, Raulf $[\mathrm{Ra}$ already studied. The approach was quite different, by reducing the problem to the estimation of the sums of special values of the Dirichlet's $L$ functions with the class number formula. While she also gave expressions of the coefficients of the leading terms, they were too complicated to be evaluated and the estimates of the reminder terms are rough. Compared to her results, our leading terms are simpler and the estimates of the reminder terms are sharper. In fact, the approximations of the coefficients are smoothly computable as described in Example of Section 4.2. The further advantage is that our approach will be arranged and extended easily. For example in Theorem 4.11, discussing only the arithmetic properties of the discriminants, we obtain the asymptotic formulas of the class number sums over fundamental discriminants in arithmetic progressions. Moreover, after overcoming some problems for the prime geodesic theorems on hyperbolic three manifolds, the results in this paper will be extended to the class number sums of the binary quadratic forms over imaginary quadratic fields.

\section{Prime geodesic theorem}

Let $H:=\{x+y \sqrt{-1} \in \mathbb{C} \mid x, y \in \mathbb{R}, y>0\}$ be the upper half plane with hyperbolic metric and $\Gamma$ a discrete subgroup of $\mathrm{SL}_{2}(\mathbb{R})$ with $\operatorname{vol}(\Gamma \backslash H)<\infty$. Denote by $\operatorname{Prim}(\Gamma)$ the set of primitive hyperbolic conjugacy classes of $\Gamma$ and $N(\gamma)$ the square of the larger eigenvalue of $\gamma \in \operatorname{Prim}(\Gamma)$. For a finite dimensional unitary representation $\chi$, it is known that

$$
\pi_{\Gamma, \chi}(x):=\sum_{\substack{\gamma \in \operatorname{Prim}(\Gamma) \\ N(\gamma)<x}} \operatorname{tr} \chi(\gamma)=\sum_{0 \leq \lambda_{j, \chi} \leq 1 / 4} \operatorname{li}\left(x^{s_{j, \chi}}\right)+R_{\Gamma, \chi}(x),
$$


where $\lambda_{j, \chi}$ is the $j$-th eigenvalue of the Laplacian acting on the sections of the flat vector bundle on $\Gamma \backslash H$ associated to the representation $\chi, s_{j, \chi}:=1 / 2+\sqrt{1 / 4-\lambda_{j, \chi}}$ and $R_{\Gamma, \chi}(x)$ is the essential reminder term for the asymptotic behavior of $\pi_{\Gamma, \chi}(x)$. Note that (2.1) is called by the prime geodesic theorem for $(\Gamma, \chi)$ and $R_{\Gamma, \chi}(x)$ is presently bounded by $R_{\Gamma, \chi}(x)=O\left(x^{3 / 4}\right)$ (see, e.g. [Se] and [He]). For the implied constant of its estimate of $R_{\Gamma, \chi}(x)$, Jorgenson and Kramer [JK] proved the following lemma.

Lemma 2.1. ([JK] and also Lemma 9.6.2 in $[B \mathrm{Bu}]$ ) Let $\Gamma$ be a discrete subgroup of $\mathrm{SL}_{2}(\mathbb{R})$ with $\operatorname{vol}(\Gamma \backslash H)<\infty$ and $\chi$ a finite dimensional unitary representation of $\Gamma$. Then, there exists a constant $A_{\Gamma}>0$ depending on $\Gamma$ such that

$$
\left|R_{\Gamma, \chi}(x)\right| \leq \operatorname{dim} \chi A_{\Gamma} x^{3 / 4}
$$

for sufficiently large $x>0$.

Let $\Gamma^{\prime}$ be a normal subgroup of $\Gamma$ with $\left[\Gamma: \Gamma^{\prime}\right]<\infty$. Denote by $G:=\Gamma / \Gamma^{\prime}$ and $\iota: \Gamma \rightarrow G$ the natural projection. According to [Sa1] and [Su], we have the following asymptotic formula for a conjugacy class $[g]$ in $G$.

$$
\pi_{\Gamma}\left(x ; \Gamma^{\prime},[g]\right):=\#\{\gamma \in \operatorname{Prim}(\Gamma) \mid \iota(\gamma) \subset[g], N(\gamma)<x\} \sim \frac{\#[g]}{\# G} \operatorname{li}(x) .
$$

This can be interpreted as an analogue of the Tchebotarev density theorem for algebraic number fields (see, e.g. $\mathrm{Ar}]$, [Ta] and [Tc]). Put

$$
R_{\Gamma}\left(x ; \Gamma^{\prime},[g]\right):=\pi_{\Gamma}\left(x ; \Gamma^{\prime},[g]\right)-\frac{\#[g]}{\# G} \operatorname{li}(x) .
$$

We now estimate $R_{\Gamma}\left(x ; \Gamma^{\prime},[g]\right)$ as follows.

Lemma 2.2. Let $\Gamma$ be a discrete subgroup of $\mathrm{SL}_{2}(\mathbb{R})$ with $\operatorname{vol}(\Gamma \backslash H)<\infty$ and $\Gamma^{\prime}$ a normal subgroup of $\Gamma$ with $\left[\Gamma: \Gamma^{\prime}\right]<\infty$. Then there exists a constant $B_{\Gamma}>0$ depending on $\Gamma$ such that

$$
\left|R_{\Gamma}\left(x ; \Gamma^{\prime},[g]\right)\right| \leq \#[g] B_{\Gamma} x^{c_{\Gamma, \Gamma^{\prime}}}
$$

for sufficiently large $x>0$, where

$$
c_{\Gamma, \Gamma^{\prime}}:=\max \left\{3 / 4, s_{j, \chi} \mid \chi \in \hat{G}, 0<\lambda_{j, \chi} \leq 1 / 4\right\} .
$$

Proof. For an element $g \in G$, it holds that

$$
\sum_{\chi \in \hat{G}} \operatorname{tr} \chi\left(g^{-1}\right) \pi_{\Gamma, \chi}(x)=\frac{\# G}{\#[g]} \pi_{\Gamma}\left(x ; \Gamma^{\prime},[g]\right),
$$

(see $[\mathrm{Su}])$. By virtue of (2.1), we have

$$
\pi_{\Gamma}\left(x ; \Gamma^{\prime},[g]\right)=\frac{\#[g]}{\# G} \sum_{\chi \in \hat{G}} \operatorname{tr} \chi\left(g^{-1}\right)\left(\sum_{0 \leq \lambda_{j, \chi} \leq 1 / 4} \operatorname{li}\left(x^{s_{j, \chi}}\right)+R_{\Gamma, \chi}(x)\right) .
$$


Put

$$
\begin{aligned}
R^{(1)}(x) & :=\frac{\#[g]}{\# G} \sum_{\chi \in \hat{G}} \operatorname{tr} \chi\left(g^{-1}\right) \sum_{0<\lambda_{j, \chi} \leq 1 / 4} \operatorname{li}\left(x^{s_{j, \chi}}\right), \\
R^{(2)}(x, T) & :=\frac{\#[g]}{\# G} \sum_{\chi \in \hat{G}} \operatorname{tr} \chi\left(g^{-1}\right) R_{\Gamma, \chi}(x) .
\end{aligned}
$$

Due to Lemma 2.1, we get

$$
\left|R^{(2)}(x, T)\right| \leq \frac{\#[g]}{\# G} A_{\Gamma} x^{3 / 4} \sum_{\chi \in \hat{G}}\left|\operatorname{tr} \chi\left(g^{-1}\right)\right| \operatorname{dim} \chi \leq \#[g] A_{\Gamma} x^{3 / 4} .
$$

Since $\#\left\{0<\lambda_{j, \chi} \leq 1 / 4\right\} \leq B_{\Gamma}^{\prime} \operatorname{dim} \chi$ for some constant $B_{\Gamma}^{\prime}>0$ depending on $\Gamma$ ([Bur], [Zo] and [JK]), we also have

$$
\left|R^{(1)}(x)\right| \leq \frac{\#[g]}{\# G} \sum_{\chi \in \hat{G}}\left|\operatorname{tr} \chi\left(g^{-1}\right)\right| \sum_{0<\lambda_{j, \chi} \leq 1 / 4} \operatorname{li}\left(x^{\max \left(s_{j, \chi}\right)}\right) \leq \#[g] B_{\Gamma}^{\prime} \operatorname{li}\left(x^{\max \left(s_{j, \chi}\right)}\right) .
$$

Thus we obtain the estimate in the lemma.

In Section 4, we will use Lemma 2.2 in the case that $\Gamma=\mathrm{SL}_{2}(\mathbb{Z})$ and $\Gamma^{\prime}$ is a principal congruence subgroup to get the main results. For such $\Gamma$ and $\Gamma^{\prime}$, it is known that $\lambda_{j, \chi}=0$ or $\lambda_{j, \chi} \geq 975 / 4096$ (see [Se], [LRS], [KS] and [Ki]). Then we can take $c_{\mathrm{SL}_{2}(\mathbb{Z}), \hat{\Gamma}(n)}=3 / 4$.

Remark that it has been proved that $R_{\Gamma}(x) \ll x^{7 / 10}$ when $\Gamma$ is a congruence subgroup of $\mathrm{SL}_{2}(\mathbb{Z})$ (see $[\mathrm{LS}],[\mathrm{LRS}]$ and also $[\mathrm{Ko}]$ ). While it is better than $R_{\Gamma}(x) \ll x^{3 / 4}$, we require the estimate with implied constants as described in Lemma 2.1 and 2.2. Of course, getting the estimate $R_{\Gamma}(x) \ll x^{7 / 10}$ with implied constants is not necessarily impossible, and it will give better results if possible. However, discussing it is exhaustive and will make this paper too heavy. Thus we will apply Lemma 2.2 in this paper.

\section{Modular group and binary quadratic forms}

\subsection{Relations between the modular group and quadratic forms}

Let

$$
Q(x, y)=[a, b, c]:=a x^{2}+b x y+c y^{2}
$$

be a binary quadratic form over $\mathbb{Z}$ with $a, b, c \in \mathbb{Z}$ and $\operatorname{gcd}(a, b, c)=1$. We call that quadratic forms $Q$ and $Q^{\prime}$ are equivalent and write $Q \sim Q^{\prime}$ if there exists $g \in \mathrm{SL}_{2}(\mathbb{Z})$ such that $Q(x, y)=Q^{\prime}((x, y) . g)$. Denote by $h(D)$ the number of equivalence classes of the quadratic forms of given $D=b^{2}-4 a c$. Let $D=D(Q):=b^{2}-4 a c$ be the discriminant of $[a, b, c]$. It is known that, if $D>0$, then there are infinitely many positive solutions $(t, u)$ of the Pell equation $t^{2}-D u^{2}=4$. Put $\left(t_{j}, u_{j}\right)=\left(t_{j}(D), u_{j}(D)\right)$ the $j$-th positive solution 
of $t^{2}-D u^{2}=4$ and $\epsilon_{j}(D):=\left(t_{j}(D)+u_{j}(D)\right) / 2$. Note that $\epsilon(D)=\epsilon_{1}(D)$ is called by the fundamental unit of $D$ in the narrow sense, and it holds that $\epsilon_{j}(D)=\epsilon(D)^{j}$.

For a quadratic form $Q=[a, b, c]$ and a solution $(t, u)$ of $t^{2}-D u^{2}=4$, we put

$$
\gamma(Q,(t, u)):=\left(\begin{array}{cc}
\frac{t+b u}{2} & -c u \\
a u & \frac{t-b u}{2}
\end{array}\right) \in \mathrm{SL}_{2}(\mathbb{Z})
$$

Conversely, for $\gamma=\left(\gamma_{i, j}\right)_{1 \leq i, j \leq 2} \in \mathrm{SL}_{2}(\mathbb{Z})$, we put

$$
\begin{aligned}
& t_{\gamma}:=\gamma_{11}+\gamma_{22}, \quad u_{\gamma}:=\operatorname{gcd}\left(\gamma_{21}, \gamma_{11}-\gamma_{22},-\gamma_{12}\right), \\
& a_{\gamma}:=\gamma_{21} / u_{\gamma}, \quad b_{\gamma}:=\left(\gamma_{11}-\gamma_{22}\right) / u_{\gamma}, \quad c_{\gamma}:=-\gamma_{12} / u_{\gamma}, \\
& Q_{\gamma}:=\left[a_{\gamma}, b_{\gamma}, c_{\gamma}\right], \quad D_{\gamma}:=\frac{t_{\gamma}^{2}-4}{u_{\gamma}^{2}}=b_{\gamma}^{2}-4 a_{\gamma} c_{\gamma} .
\end{aligned}
$$

It is known that (3.1) and (3.2) gives a one-to-one correspondence between equivalence classes of primitive binary quadratic forms $D>0$ and the elements of $\operatorname{Prim}\left(\mathrm{SL}_{2}(\mathbb{Z})\right)$. We now note the following elementary facts without proof (see, e.g. [G]).

Fact 3.1. Suppose that $\gamma, \gamma_{1}, \gamma_{2} \in \mathrm{SL}_{2}(\mathbb{Z})$ are not in any finite group in $\mathrm{SL}_{2}(\mathbb{Z})$. Then we have

1. $\gamma\left(Q_{\gamma},\left(t_{\gamma}, u_{\gamma}\right)\right)=\gamma$.

2. $D(Q)=D_{\gamma(Q,(t, u))}$ for any $t, u \geq 1$ with $t^{2}-D u^{2}=4$, and $D_{\gamma}=D\left(Q_{\gamma}\right)$.

3. $\left(t_{\gamma(Q,(t, u))}, u_{\gamma(Q,(t, u))}\right)=(t, u)$, and $\left(t_{\gamma}, u_{\gamma}\right)$ coincides one of $\left(t_{j}(D), u_{j}(D)\right)$.

4. If $Q_{\gamma_{1}}=Q_{\gamma_{2}}$ then $Q_{\gamma_{1} \gamma_{2}}=Q_{\gamma_{1}}=Q_{\gamma_{2}}$, and $\left(t_{\gamma_{1} \gamma_{2}}, u_{\gamma_{1} \gamma_{2}}\right)=\left(\frac{1}{2}\left(t_{\gamma_{1}} t_{\gamma_{2}}+D u_{\gamma_{1}} u_{\gamma_{2}}\right), \frac{1}{2}\left(t_{\gamma_{1}} u_{\gamma_{2}}+\right.\right.$ $\left.\left.t_{\gamma_{2}} u_{\gamma_{1}}\right)\right)$.

5. $Q_{g^{-1} \gamma g}(x, y)=Q_{\gamma}((x, y) . g)$ for any $g \in \mathrm{SL}_{2}(\mathbb{Z})$.

Since $N(\gamma)$ for $\gamma \in \operatorname{Prim}\left(\mathrm{SL}_{2}(\mathbb{Z})\right)$ coincides $\epsilon_{1}(D)^{2}$ of corresponding quadratic form, the prime geodesic theorem for $\mathrm{SL}_{2}(\mathbb{Z})$ yields the following asymptotic formula.

$$
\pi_{\mathrm{SL}_{2}(\mathbb{Z})}\left(x^{2}\right)=\sum_{\substack{D \in \mathfrak{D} \\ \epsilon(D)<x}} h(D)=\operatorname{li}\left(x^{2}\right)+R_{\mathrm{SL}_{2}(\mathbb{Z})}\left(x^{2}\right)
$$

where $\mathfrak{D}:=\{D>0 \mid D \equiv 0,1 \bmod 4$, not square $\}$.

\subsection{On $\mathrm{PSL}_{2}(\mathbb{Z} / n \mathbb{Z})$}

First we prepare some notations.

For integers $n, m \geq 1, m \| n$ means that $m \mid n$ and $\operatorname{gcd}(m, n / m)=1$, and $n_{m}$ denotes the maximal divisor of $n$ relatively prime to $m$. For an integer $n \geq 1$, let

$$
\begin{aligned}
\mathbb{Z}_{n} & :=\mathbb{Z} / n \mathbb{Z}, \quad \mathbb{Z}_{n}^{(2)}:=\mathbb{Z}_{n}^{*} /\left(\mathbb{Z}_{n}^{*}\right)^{2}, \\
\hat{\Gamma}(n) & :=\operatorname{Ker}\left(\mathrm{SL}_{2}(\mathbb{Z}) \stackrel{\text { proj. }}{\rightarrow} \operatorname{PSL}_{2}\left(\mathbb{Z}_{n}\right)\right) \\
& =\left\{\gamma \in \mathrm{SL}_{2}(\mathbb{Z}) \mid \gamma \equiv \alpha I \bmod n, \alpha^{2} \equiv 1 \bmod n\right\} .
\end{aligned}
$$


The subgroup $\hat{\Gamma}(n)$ of $\mathrm{SL}_{2}(\mathbb{Z})$ is called by the principle congruence subgroup of level $n$. If the factorization of $n$ is $n=\prod_{p} p^{r}$ then

$$
\begin{aligned}
& \hat{\Gamma}(n)=\bigcap_{p \mid n} \hat{\Gamma}\left(p^{r}\right), \\
& v(n):=\left[\mathrm{SL}_{2}(\mathbb{Z}): \hat{\Gamma}(n)\right]=\# \mathrm{PSL}_{2}\left(\mathbb{Z}_{n}\right)=\prod_{p \mid n} \frac{p^{3 r-2}\left(p^{2}-1\right)}{\# \mathbb{Z}_{p^{r}}^{(2)}} .
\end{aligned}
$$

Note that (see, e.g. G])

$$
\# \mathbb{Z}_{p^{r}}^{(2)}= \begin{cases}1, & \left(p^{r}=2\right) \\ 2, & \left(p^{r}=4 \text { or } 2 \nmid p\right), \\ 4, & (p=2, r \geq 3) .\end{cases}
$$

We now prepare the following lemma.

Lemma 3.1. Let $n \geq 1$ be an integer and $\gamma \in \mathrm{SL}_{2}(\mathbb{Z})$. Then $\gamma \in \hat{\Gamma}(n)$ if and only if $n \mid u_{\gamma}$.

The proof for prime $n$ was already done in [Sa1], and its generalization to composite $n$ is similar.

Let $p$ be a prime and $k, r \geq 1$ integers. Due to (3.1) and Lemma 3.1, we can express the element $\gamma \in \hat{\Gamma}\left(p^{k}\right)-\hat{\Gamma}\left(p^{k+1}\right)$ in $\mathrm{PSL}_{2}\left(\mathbb{Z}_{p^{r+k}}\right)$ by

$$
\gamma \equiv\left(\begin{array}{cc}
\frac{T+B p^{k}}{2} & A p^{k} \\
-C p^{k} & \frac{T-B p^{k}}{2}
\end{array}\right) \bmod p^{r+k},
$$

where $A \equiv a_{\gamma}\left(u_{\gamma} / p^{k}\right), B \equiv b_{\gamma}\left(u_{\gamma} / p^{k}\right), C \equiv c_{\gamma}\left(u_{\gamma} / p^{k}\right) \bmod p^{r}$ and $T \equiv t_{\gamma} \bmod p^{r+k}$. Note that $p^{k} \| u_{\gamma}$ and $T$ satisfies

$$
T^{2} \equiv 4+\left(B^{2}-4 A C\right) p^{2 k} \bmod \begin{cases}2^{r+k+2} & (p=2) \\ p^{r+k} & (p \geq 3)\end{cases}
$$

Let $u_{n}:=\left(u_{\gamma}\right)_{n}$ and $D(n)=\left(D_{\gamma}\right)(n):=u_{n}^{2} D_{\gamma}$. We see that $D(p)=B^{2}-4 A C$.

We now prove the following proposition as a preparation for the main theorem.

Proposition 3.2. Let $r, k$ be integers, $p$ a prime number and $\delta \in \mathbb{Z}_{p^{r}}$. Put

$$
\begin{aligned}
& \mathcal{T}\left(\delta ; p^{r}, k\right):=\#\left\{T \in \mathbb{Z}_{p^{r+k}} \mid T^{2} \equiv 4+\delta p^{2 k} \bmod \left\{\begin{array}{ll}
2^{r+k+2} & (p=2) \\
p^{r+k} & (p \geq 3)
\end{array}\right\},\right. \\
& \mathcal{A}\left(\delta ; p^{r}\right):=\#\left\{(A, B, C) \in \mathbb{Z}_{p^{r}}^{3} \mid p \nmid \operatorname{gcd}(A, B, C), B^{2}-4 A C \equiv \delta \bmod p^{r}\right\}, \\
& \hat{\Gamma}\left(\delta ; p^{r}, k\right):=\left\{\gamma \in \mathrm{PSL}_{2}\left(\mathbb{Z}_{p^{r+k}}\right) \mid D(p) \equiv \delta \bmod p^{r}\right\} .
\end{aligned}
$$

Then it holds that

$$
\# \hat{\Gamma}\left(\delta ; p^{r}, k\right)=\mathcal{T}\left(\delta ; p^{r}, k\right) \mathcal{A}\left(\delta ; p^{r}\right) / \# \mathbb{Z}_{p^{r}}^{(2)}
$$


Furthermore, $\mathcal{T}\left(\delta ; p^{r}, k\right)$ and $\mathcal{A}\left(\delta ; p^{r}\right)$ are given as follows.

$$
\begin{aligned}
& \mathcal{T}\left(\delta ; 2^{r}, k\right)= \begin{cases}4, & \left(32 \mid 2^{2 k} \delta\right), \\
2^{l+1}, & \left(k=0, \delta \equiv-4+2^{2 l} \alpha^{2} \bmod 2^{r+2}, 0 \leq l \leq r / 2\right), \\
2^{[r / 2]}, & \left(k=0, \delta \equiv-4 \bmod 2^{r+2},\right. \\
0, & \text { or } \left.k=0, \delta \equiv-4+2^{r+1} \bmod 2^{r+2}, r \text { is odd }\right),\end{cases} \\
& \mathcal{T}\left(\delta ; p^{r}, k\right)= \begin{cases}2, & \left(p \mid \delta p^{2 k}\right), \\
2 p^{l}, & \left(k=0, \delta \equiv-4+\alpha^{2} p^{2 l} \bmod p^{r}, 0 \leq l<r / 2\right), \\
p^{[r / 2]}, & \left(k=0, \delta \equiv-4 \bmod p^{r}\right), \\
0, & (\text { otherwise }),\end{cases} \\
& \mathcal{A}\left(\delta ; 2^{r+2}\right)= \begin{cases}3 \times 2^{2 r-2}, & (\delta \equiv 1 \bmod 8), \\
2^{2 r-2}, & (\delta \equiv 5 \bmod 8), \\
3 \times 2^{2 r-3}, & (4 \mid \delta), \\
0, & (\text { otherwise }),\end{cases} \\
& \mathcal{A}\left(\delta ; p^{r}\right)= \begin{cases}p^{2 r-1}(p+1), & ((\delta / p)=1), \\
p^{2 r-1}(p-1), & ((\delta / p)=-1), \\
p^{2 r-2}\left(p^{2}-1\right), & (p \mid \delta),\end{cases}
\end{aligned}
$$

where $p$ is an odd prime and $\alpha \in \mathbb{Z}_{2^{r+2}}^{*}$ or $\mathbb{Z}_{p^{r}}^{*}$.

Proof. We first note that $\mathcal{T}\left(\delta ; p^{r}, k\right)$ does not depend on $(A, B, C)$ but $\delta$. Then we have

$$
\# \hat{\Gamma}\left(\delta ; p^{r}, k\right)=\mathcal{T}\left(\delta ; p^{r}, k\right) \mathcal{A}\left(\delta ; p^{r}\right) / \# \mathbb{Z}_{p^{r}}^{(2)}
$$

Next, compute $\mathcal{T}\left(\delta ; p^{r}, k\right)$. The equation is

$$
T^{2} \equiv 4+\delta p^{2 k} \bmod \left\{\begin{array}{ll}
2^{r+k+2} & (p=2) \\
p^{r+k} & (p \geq 3)
\end{array} .\right.
$$

It is easy to see that this has solutions if and only if $4+\delta p^{2 k} \equiv p^{2 l} \alpha^{2}$ or $\equiv 0$ for some $l \geq 0$ and $\alpha \in \mathbb{Z}_{p^{r}}^{*}$. The solutions of $T^{2} \equiv \alpha^{2} p^{2 l} \bmod p^{r}$ are written in the form $T \equiv \pm \alpha p^{l}+\beta p^{r-l}$ for $\beta \in \mathbb{Z}_{p^{l}}$ when $p \geq 3$, and are $T \equiv \omega \alpha p^{l}+\beta 2^{r-l-1}$ for $\omega \in \mathbb{Z}_{p^{r}}^{(2)}$ and $\beta \in \mathbb{Z}_{p^{l+1}}$ when $p=2$. On the other hand, the solutions of $T^{2} \equiv 0 \bmod p^{r}$ are in the form $T \equiv \beta p^{l}$ with $2 l \geq r$. Thus we can get $\mathcal{T}\left(\delta ; p^{r}, k\right)$ as described in the proposition.

Finally, we compute $\mathcal{A}\left(\delta ; p^{r}\right)$.

The case of $\delta \equiv 5 \bmod 8, p=2$. In this case, $B$ must be odd and the number of such $B$ is $2^{r-1}$. Since $\left(\delta-B^{2}\right) / 4$ is odd, $C$ is given by $C \equiv A^{-1} \frac{\delta-B^{2}}{4}$ for given $A \in \mathbb{Z}_{2^{r}}^{*}$ and $B$. Thus we have $\mathcal{A}\left(\delta ; 2^{r+2}\right)=2^{r-1} \times 2^{r-1}=2^{2 r-2}$.

The case of $4 \mid \delta, p=2$. When $4 \mid \delta, B$ must be even. If $\left(\delta-B^{2}\right) / 4 \not \equiv 0 \bmod 2$, then $C$ is determined by $C \equiv A^{-1} \frac{\delta-B^{2}}{4}$ for given $A \in \mathbb{Z}_{2^{r}}^{*}$ and $B$. Then the number of such $(A, B, C)$ is $2^{r-2} \times 2^{r-1}=2^{2 r-3}$. If $\left(\delta-B^{2}\right) / 4 \equiv 0 \bmod 2$, then $2 \mid A C$. Since $2 \nmid \operatorname{gcd}(A, B, C)$, one of 
$A, C$ is odd. This means that the number of such $(A, B, C)$ is $2^{r-2} \times 2 \times 2^{r-1}=2^{2 r-2}$. Thus we have $\mathcal{A}\left(\delta ; 2^{r+2}\right)=2^{2 r-3}+2^{2 r-2}=3 \times 2^{2 r-3}$.

The case of $\delta \equiv 1 \bmod 8, p=2$. In this case, $B$ must be odd and $A C$ even. Putting $B=1+2 B_{1}$ and $\delta=1+8 \delta_{1}$ into the equation $B^{2}-4 A C \equiv \delta \bmod 2^{r+2}$, we have

$$
B_{1}^{2}+B_{1} \equiv \delta_{1}+\frac{A C}{2} \bmod 2^{r-1}
$$

If the right hand side of the above is even, then there are two $B_{1} \in \mathbb{Z}_{2^{r-1}}$, and if it is odd then there are no such $B_{1}$. It is easy to see that the number of $(A, C)$ with $2 \mid A C$ and $2 \mid \frac{A C}{2}-\delta_{1}$ is $3 \times 2^{2 r-3}$. Thus we have $\mathcal{A}\left(\delta ; 2^{r+2}\right)=3 \times 2^{2 r-3} \times 2=3 \times 2^{2 r-2}$.

The case of $(\delta / p)=-1, p \geq 3$. When $(\delta / p)=-1$, we have $\delta-B^{2}=4 A C \not \equiv 0 \bmod p$ for any $B \in \mathbb{Z}_{p^{r}}$. Then $C$ is uniquely determined by $C \equiv(4 A)^{-1}\left(\delta-B^{2}\right)$ for given $B \in \mathbb{Z}_{p^{r}}$ and $A \in \mathbb{Z}_{p^{r}}^{*}$. Thus we have $\mathcal{A}\left(\delta ; p^{r}\right)=p^{r} \times p^{r-1}(p-1)=p^{2 r-1}(p-1)$.

The case of $p \mid \delta, p \geq 3$. When $p \nmid B, C$ is uniquely determined by $C \equiv(4 A)^{-1}(\delta-$ $\left.B^{2}\right) \bmod p^{r}$ for given $A \in \mathbb{Z}_{p^{r}}^{*}$. Then the number of such $(A, B, C)$ is $p^{r-1}(p-1) \times p^{r-1}(p-1)=$ $p^{2 r-2}(p-1)^{2}$. When $p \mid B$, we have $p \mid A C$. Since $p \nmid \operatorname{gcd}(A, B, C)$, one of $A, C$ is not divided by $p$. Then the number of such $(A, B, C)$ is $p^{r-1} \times 2 \times p^{r-1}(p-1)=2 p^{2 r-2}(p-1)$. Thus we have $\mathcal{A}\left(\delta ; p^{r}\right)=p^{2 r-2}(p-1)^{2}+2 p^{2 r-2}(p-1)=p^{2 r-2}\left(p^{2}-1\right)$.

The case of $(\delta / p)=1, p \geq 3$. When $(\delta / p)=1$, there are $B \in \mathbb{Z}_{p^{r}}$ such that $p \mid \delta-B^{2}$. Since the number of $B \in \mathbb{Z}_{p^{r}}$ with $B^{2} \equiv \delta \bmod p^{l}(1 \leq l \leq r)$ is $2 p^{r-l}$, the number of $B \in \mathbb{Z}_{p^{r}}$ with $p^{l} \| \delta-B^{2}$ is $p^{r-1}(p-2)$ for $l=0,2 p^{r-l-1}(p-1)$ for $1 \leq l \leq r-1$ and 2 for $l=r$.

When $p \nmid \delta-B^{2}, C$ is uniquely determined by $C \equiv(4 A)^{-1}\left(\delta-B^{2}\right)$ for given $A \in \mathbb{Z}_{p^{r}}^{*}$. Then the number of such $(A, C)$ is $p^{r-1}(p-1)$. When $\delta-B^{2} \equiv \alpha p^{l}$ for $1 \leq l \leq r-1$ and $\alpha \in \mathbb{Z}_{p^{r-l}}^{*}, A, C$ are given by $A \equiv a_{1} p^{l_{1}}$ and $C \equiv c_{1} p^{l-l_{1}}$ for $0 \leq l_{1} \leq l, a_{1} \in \mathbb{Z}_{p^{r-l}}^{*}$ and $c_{1} \in \mathbb{Z}_{p^{r+l_{1}-l}}^{*}$. Since $a_{1} c_{1} \equiv \alpha \bmod p^{r-l}$, we see that the number of such $\left(a_{1}, c_{1}\right)$ is $p^{r-1}(p-1)$ for given $l$ and $l_{1}$. When $p^{r} \mid \delta-B^{2}, A$ and $C$ are given by $A \equiv a_{1} p^{l_{1}}$ and $C \equiv c_{1} p^{r-l_{1}}$ for $0 \leq l_{1} \leq r, a_{1} \in \mathbb{Z}_{p^{r-l_{1}}}^{*}$ and $c_{1} \in \mathbb{Z}_{p^{l_{1}}}$. Then the number of such $(A, C)$ is

$$
\sum_{0 \leq l_{1} \leq r-1} p^{r-l_{1}-1}(p-1) \times p^{l_{1}}+p^{r}=(r+1) p^{r}-r p^{r-1} .
$$

Thus we get

$$
\begin{aligned}
\mathcal{A}\left(\delta ; p^{r}\right)= & p^{r-1}(p-2) p^{r-1}(p-1)+\sum_{1 \leq l \leq r-1} 2 p^{r-l-1}(p-1)(l+1) p^{r-1}(p-1) \\
& +2\left((r+1) p^{r}-r p^{r-1}\right) \\
= & p^{2 r-1}(p+1) .
\end{aligned}
$$

\section{Main results}

Let $\mathcal{C}$ be a "condition" for $D \in \mathfrak{D}$ and $\mathfrak{D}(\mathcal{C})$ the set of $D \in \mathfrak{D}$ satisfying the condition $\mathcal{C}$. For example, $\mathfrak{D}(D \equiv 1 \bmod 4)$ is the set of $D \in \mathfrak{D}$ with $D \equiv 0,1 \bmod 4$. For a condition $\mathcal{C}$, 
denote by

$$
\pi(x ; \mathcal{C}):=\sum_{\substack{D \in \mathfrak{D}(\mathcal{C}) \\ \epsilon(D)<x}} h(D)
$$

If $\pi(x, \mathcal{C}) / \operatorname{li}\left(x^{2}\right)$ converges as $x \rightarrow \infty$, put

$$
\begin{aligned}
\eta(\mathcal{C}) & :=\lim _{x \rightarrow \infty} \frac{\pi(x, \mathcal{C})}{\operatorname{li}\left(x^{2}\right)} \\
R(x ; \mathcal{C}) & :=\pi(x, \mathcal{C})-\eta(\mathcal{C}) \operatorname{li}\left(x^{2}\right)
\end{aligned}
$$

The aim in this section is to study $\pi(x ; \mathcal{C})$ when $\mathcal{C}$ is given by the arithmetic progressions.

\subsection{Lemmas}

Lemma 4.1. Let $n_{1}, n_{2} \geq 1$ be integers and $\delta \in \mathbb{Z}_{n_{1}}$. Then $\eta\left(D_{n_{1}} \equiv \delta \bmod n_{1}, n_{2} \mid u\right)$ exists and

$$
\begin{aligned}
& \left|R\left(x ; D\left(n_{1}\right) \equiv \delta \bmod n_{1}, n_{2} \mid u\right)\right| \\
& \leq \eta\left(D\left(n_{1}\right) \equiv \delta \bmod n_{1}, n_{2} \mid u\right) v\left(n_{1} n_{2}\right) B_{\mathrm{SL}_{2}(\mathbb{Z})} x^{3 / 2} .
\end{aligned}
$$

Proof. Due to Fact 3.1, we see that the element

$$
\gamma \equiv\left(\begin{array}{cc}
\frac{t+B n_{2}}{2} & A n_{2} \\
-C n_{2} & \frac{t-B n_{2}}{2}
\end{array}\right) \bmod n_{1} n_{2}
$$

with $\delta \equiv B^{2}-4 A C \bmod n_{1}$ and $n_{1} \nmid \operatorname{gcd}(A, B, C)$ corresponds to the quadratic form with $D\left(n_{1}\right) \equiv \delta \bmod n_{1}$ and $n_{2} \mid u$. Then, according to Lemma 2.2 , we have

$$
\begin{aligned}
& \pi\left(x ; D\left(n_{1}\right) \equiv \delta \bmod n_{1}, n_{2} \mid u\right) \\
& =\sum_{\substack{[g] \in \operatorname{Conj}\left(\operatorname{SL}_{2}(\mathbb{Z})\right) \\
\left(D_{g}\right)\left(n_{1}\right) \equiv \delta \bmod n_{1}, n_{2} \mid u_{g}}} \#[g] \pi_{\mathrm{SL}_{2}(\mathbb{Z})}\left(x ; \hat{\Gamma}\left(n_{1} n_{2}\right),[g]\right) .
\end{aligned}
$$

Thus we get

$$
\begin{aligned}
& \eta\left(D\left(n_{1}\right) \equiv \delta \bmod n_{1}, n_{2} \mid u\right)=\sum_{[g]} \frac{\#[g]}{v\left(n_{1} n_{2}\right)}, \\
& \left|R\left(x ; D\left(n_{1}\right) \equiv \delta \bmod n_{1}, n_{2} \mid u\right)\right|=\left|\sum_{[g]} R_{\mathrm{SL}_{2}(\mathbb{Z})}\left(x^{2} ; \hat{\Gamma}\left(n_{1} n_{2}\right),[g]\right)\right| \leq \sum_{[g]} \#[g] B_{\mathrm{SL}_{2}(\mathbb{Z})} x^{3 / 2},
\end{aligned}
$$

and the lemma follows immediately.

When $n_{1}, n_{2}$ are powers of the same prime $p$, we can easily find the following lemma. 
Lemma 4.2. Let $p$ be a prime number, $k \geq 0, r \geq 1$ integers and $\delta \in \mathbb{Z}_{p^{r}}$. Then we have

$$
\eta\left(D(p) \equiv \delta \bmod p^{r}, p^{k} \| u\right)=\frac{\# \hat{\Gamma}\left(\delta, p^{r}, k\right)}{v\left(p^{r+k}\right)},
$$

where $\# \hat{\Gamma}\left(\delta, p^{r}, k\right)$ is explicitly calculated in Proposition 3.2 .

For composite $n_{1}$, we give the following lemmas.

Lemma 4.3. Let $n_{1}, n_{2} \geq 1$ be integers relatively prime to each other and fix $[g]$ a conjugacy class in $\mathrm{PSL}_{2}\left(\mathbb{Z}_{n_{1} n_{2}}\right)$. Then we have

$$
\#[g]=\#[g]_{n_{1}} \#[g]_{n_{2}},
$$

where $[g]_{n_{1}},[g]_{n_{2}}$ are the sets of $g_{1} \in \mathrm{PSL}_{2}\left(\mathbb{Z}_{n_{1}}\right)$ with $g_{1} \equiv g \bmod n_{1}$ and $g_{2} \in \mathrm{PSL}_{2}\left(\mathbb{Z}_{n_{2}}\right)$ with $g_{2} \equiv g \bmod n_{2}$ for some $g \in[g]$ respectively.

Proof. See the discussions in the proof of Proposition 4.5 in [HW].

Lemma 4.4. Let $n_{1}, n_{2} \geq 1$ be integers and $n_{1}=p_{1}^{r_{1}} \cdots p_{l}^{r_{l}}$ the factorization of $n_{1}$. Denote by $m_{1}$ the maximal divisor of $n_{2}$ relatively prime to $n_{1}$, namely $n_{2}=p_{1}^{k_{1}} \cdots p_{l}^{k_{l}} m_{1}$ for some $k_{1}, \cdots, k_{l} \geq 0$ and relatively prime $m_{1}, n_{1} \geq 1$. Then we have

$$
\begin{aligned}
& \eta\left(D\left(n_{1}\right) \equiv \delta \bmod n_{1}, n_{2} \mid u\right) \\
& =\eta\left(m_{1} \mid u\right) \prod_{i=1}^{l} \eta\left(D\left(p_{i}\right) \equiv\left(n_{1}\right)_{p_{i}}^{2} \delta \bmod p^{r_{i}}, p_{i}^{k_{i}} \mid u\right) .
\end{aligned}
$$

Proof. We first note that the condition " $D\left(n_{1}\right) \equiv \delta \bmod n_{1}, n_{2} \mid u$ " is equivalent that " $D\left(p_{i}\right) \equiv\left(n_{1}\right)_{p_{i}}^{2} \delta \bmod p^{r_{i}}, p_{i}^{k_{i}} \mid u$ for any $1 \leq i \leq l$ and $m_{1} \mid u$ ". As discussed in the proofs of Lemma 4.1 and 3.1, it holds that

$$
\begin{aligned}
& \eta\left(D\left(n_{1}\right) \equiv \delta \bmod n_{1}, n_{2} \mid u\right)=\sum_{\substack{[g] \in \operatorname{Conj}\left(\mathrm{PSL}_{2}\left(\mathbb{Z}_{\left.\left.n_{1} n_{2}\right)\right)}\right) \\
\left(D_{g}\right)\left(n_{1}\right) \equiv \delta \bmod n_{1}, n_{2} \mid u_{g}\right.}} \frac{\#[g]}{v\left(n_{1} n_{2}\right)} \\
& \eta\left(D\left(p_{i}\right) \equiv\left(n_{1}\right)_{p_{i}}^{2} \delta \bmod p^{r_{i}}, p_{i}^{k_{i}} \mid u\right)=\sum_{\substack{[g] \in \operatorname{Conj}\left(\mathrm { PSL } _ { 2 } \left(\mathbb{Z}_{\left.\left.n_{1} n_{2}\right)\right)} \\
\left(D_{g}\right)\left(p_{i}\right) \equiv\left(n_{1}\right)_{p_{i}}^{2} \delta \bmod p_{i}^{r_{i}} \\
p_{i}^{k_{i}} \mid u_{g}\right.\right.}} \frac{\#[g]}{v\left(p_{i}^{r_{i}+k_{i}}\right)} \\
& \eta\left(m_{1} \mid u\right)=\frac{1}{v\left(m_{1}\right)} .
\end{aligned}
$$

Then the desired result can follow easily from Lemma 4.3.

We now prove the following proposition. This is important to prove the main theorems. 
Proposition 4.5. Let $p$ be a prime number, $r \geq 1$ an integer and $\delta \in \mathbb{Z}_{p^{r}}$. Then $\eta(D(p) \equiv$ $\left.\delta \bmod p^{r}\right)$ exists with

$$
\eta\left(D(p) \equiv \delta \bmod p^{r}\right)=\sum_{k=0}^{\infty} \eta\left(D(p) \equiv \delta \bmod p^{r}, p^{k} \| u\right)=\sum_{k=0}^{\infty} \frac{\# \hat{\Gamma}\left(\delta, p^{r}, k\right)}{v\left(p^{r+k}\right)}
$$

and the reminder term is estimated by

$$
\left|R\left(x ; D(p) \equiv \delta \bmod p^{r}\right)\right| \leq \sum_{k=0}^{\left\lfloor\log _{p} x\right\rfloor} \# \hat{\Gamma}\left(\delta, p^{r}, k\right) B_{\mathrm{SL}_{2}(\mathbb{Z})} x^{3 / 2}
$$

Proof. Since $u^{2}$ is a divisor of $t^{2}-4$, we have $u<t<\epsilon(D)+1$. Then we see that

$$
\pi\left(x ; D(p) \equiv \delta \bmod p^{r}\right)=\sum_{k \geq 0, p^{k}<x} \pi\left(x ; D(p) \equiv \delta \bmod p^{r}, p^{k} \| u\right) .
$$

Divide the right hand side of the above as follows.

$$
\sum_{k}\left(\eta(*) \operatorname{li}\left(x^{2}\right)+R(*)\right)=: L_{1}(x) \operatorname{li}\left(x^{2}\right)+L_{2}(x) .
$$

Since $\eta\left(D(p) \equiv \delta \bmod p^{r}, p^{k} \| u\right) \leq \eta\left(p^{k} \mid u\right)=v\left(p^{k}\right)^{-1} \leq C p^{-3 k}$, we have

$$
L_{1}(x)=\lim _{x \rightarrow \infty} L_{1}(x)+O\left(x^{-3}\right) .
$$

The estimation of $R\left(x ; D(p) \equiv \delta \bmod p^{r}, p^{k} \| u\right)$ directly follows from Lemma 4.1. Thus we can get the desired result.

Combining Proposition 3.2 and 4.5 , we can get the explicit values of $\eta\left(D(p) \equiv \delta \bmod p^{r}\right)$ as follows.

Corollary 4.6. Let $p$ be a prime, $r \geq 1$ an integer and $\delta \in \mathbb{Z}_{p^{r}}$. Then $\eta\left(D(p) \equiv \delta \bmod p^{r}\right)$ is described as follows.

The case of $p=2$.

$$
\begin{aligned}
& \eta\left(D(2) \equiv \delta \bmod 2^{r+2}\right)=\frac{1}{7 \times 2^{r+4}} \\
& \quad \times \begin{cases}1, & (\delta \equiv 1 \bmod 8), \\
75, & (\delta \equiv 5 \bmod 8), \\
4+7 \times 2^{l+4}, & \left(\delta \equiv-4+\alpha^{2} 2^{2 l} \bmod 2^{r+2}, 2 \leq l<r / 2\right), \\
4+7 \times 2^{[r / 2]+3}, & \left(\delta \equiv-4 \bmod 2^{r+2}, \text { or } 2 \nmid r, \delta \equiv-4+2^{r+1} \bmod 2^{r+2}\right), \\
4, & (4 \| \delta, \text { otherwise }), \\
2^{5}, & (8 \| \delta \text { or } 16 \| \delta), \\
2^{8}, & (32 \mid \delta), \\
0, & (\text { otherwise }) .\end{cases}
\end{aligned}
$$


The case of $p \geq 3$.

$$
\begin{aligned}
& \eta\left(D(p) \equiv \delta \bmod p^{r}\right)=\frac{1}{p^{r-1}\left(p^{2}-1\right)\left(p^{3}-1\right)} \\
& \times \begin{cases}\left(2+p^{[r / 2]}\left(p^{3}-1\right)\right)\left(p+\left(\frac{\delta}{p}\right)\right), & \left(p \nmid \delta, \delta \equiv-4 \bmod p^{r}\right), \\
2\left(1+p^{l}\left(p^{3}-1\right)\right)\left(p+\left(\frac{\delta}{p}\right)\right), & \left(p \nmid \delta, \delta \equiv-4+\alpha^{2} p^{2 l} \bmod p^{r},\right. \\
2\left(p+\left(\frac{\delta}{p}\right)\right), & 0 \leq l<r / 2), \\
2 p^{2}\left(p^{2}-1\right), & (p \nmid \delta, \text { otherwise }),\end{cases}
\end{aligned}
$$

We further prepare the following lemma. This will be used to estimate the reminder terms in the main theorems.

Lemma 4.7. Let $x, T>0$ be large numbers with $T \ll x$. Then we have

$$
\begin{aligned}
\pi(x ; m \mid u \text { for } m>T) & =O\left(x^{2} T^{-2+\epsilon}\right), \\
\pi\left(x ; m^{2} \mid D \text { for } m>T\right) & =O\left(x^{2} T^{-1+\epsilon}\right)
\end{aligned}
$$

for any $\epsilon>0$, where the implied constants depend on $\epsilon$.

Proof. It is known (see, e.g. [Mo]) that there exists a constant $C>0$ such that

$$
h(D) \leq C \frac{\sqrt{D} \log D}{\log \epsilon(D)} .
$$

Then, according to Fact 3.1, we get

$$
\pi(x ; m \mid u \text { for } m>T)<C \sum_{m>T} \sum_{\substack{u \geq m \\ m \mid u}} \sum_{\substack{3 \leq t \leq x \\ u^{2} \mid t^{2}-4}} \sqrt{\frac{t^{2}-4}{u^{2}}} \frac{\log \frac{t^{2}-4}{u^{2}}}{\log t}<C \sum_{m>T} \sum_{\substack{u \geq m \\ m \mid u}} u^{-1} \sum_{\substack{3 \leq t \leq x \\ u^{2} \mid t^{2}-4}} t .
$$

Since $u^{2} \mid t^{2}-4$ is equivalent that $t \equiv \pm 2 \bmod p^{2 k}$ for any $p^{k} \| u$, we have

$$
\pi(x ; m \mid u \text { for } m>T)<C \sum_{m>T} \sum_{\substack{u \geq m \\ m \mid u}} \frac{2^{\omega(u)}}{u^{3}} x^{2},
$$

where $\omega(u)$ is the number of distinct prime factors of $u$. Thus the estimate

$$
\sum_{u<Y} 2^{\omega(u)}=O\left(Y^{\epsilon}\right)
$$


due to [HarWr], gives

$$
\pi(x ; m \mid u \text { for } m>T)<C \sum_{m>T} \sum_{u} \frac{2^{\omega(u)}}{m^{3} u^{3}} x^{2}<C \sum_{m>T} \frac{2^{\omega(m)}}{m^{3-\epsilon}} x^{2}=O\left(x^{2} T^{-2+\epsilon}\right) .
$$

This completes the first estimation in the lemma.

Similarly, we see that

$$
\pi\left(x ; m^{2} \mid D \text { for } m>V\right)<\sum_{m>V} \sum_{u<x / m} \frac{1}{u} \sum_{\substack{t<x \\ t^{2}-4 \mid m^{2} u^{2}}} t<\sum_{m>V} \sum_{u<x / m} \frac{2^{\omega(m u)}}{u^{3} m^{2}} x^{2} .
$$

Then we can get the second estimate due to (4.1).

\subsection{Main theorem}

The main result in this paper is as follows.

Theorem 4.8. Let $n \geq 1$ be an integer with the factorization $n=\prod_{p \mid n} p^{r}$ and $\delta \in \mathbb{Z}_{n}$. For $\alpha \in \mathbb{Z}_{n}^{*}$, denote by

$$
W(\alpha ; n):=\xi(n) \sum_{m \geq 1, m \equiv \alpha \bmod n} \beta(m)
$$

where

$$
\begin{aligned}
\xi(n):=\prod_{p \nmid n}\left(1-v(p)^{-1}\right)=\prod_{p \nmid n, p \neq 2}\left(1-\frac{2}{p\left(p^{2}-1\right)}\right) \times \begin{cases}\frac{5}{6}, & (2 \nmid 2), \\
1, & (n \mid 2),\end{cases} \\
\beta(m):=\prod_{p \mid m} \frac{v\left(p^{l}\right)^{-1}-v\left(p^{l+1}\right)^{-1}}{1-v(p)^{-1}}=\prod_{p \mid m, p \neq 2} 2 p^{-3 l} \frac{p^{3}-1}{p^{3}-p-2} \times \begin{cases}1, & (e=0), \\
\frac{3}{20}, & (e=1), \\
\frac{3}{80}, & (e=2), \\
\frac{7}{5 \times 2^{3 e-2}}, & (e \geq 3),\end{cases}
\end{aligned}
$$

for $m=2^{e} \prod_{p \mid m, p \neq 2} p^{l}$. Then $\eta(D \equiv \delta \bmod n)$ exists and satisfies that

$$
\eta(D \equiv \delta \bmod n)=\sum_{\alpha \in \mathbb{Z}_{n}^{*}} W(\alpha ; n) \prod_{p \mid n} \eta\left(D(p) \equiv \delta \alpha^{2} \bmod p^{r}\right) .
$$

The reminder term is estimated by

$$
R(x ; D \equiv \delta \bmod n)=O\left(x^{5 / 3+\epsilon}\right)
$$

for any $\epsilon>0$, where the implied constant depends on $n$ and $\epsilon$. 
Remark 4.9. It is easy to see that

$$
\sum_{\alpha \in \mathbb{Z}_{n}^{*}} W(\alpha ; n)=1
$$

Then it holds that

$$
\eta(D \equiv \delta \bmod n)=\prod_{p \mid n} \eta\left(D(p) \equiv \delta \bmod p^{r}\right)
$$

if the value

$$
\prod_{p \mid n} \eta\left(D(p) \equiv \delta \alpha^{2} \bmod p^{r}\right)
$$

does not depend on $\alpha \in \mathbb{Z}_{n}^{*}$. For example, we have

$$
\eta(n \mid D)=\prod_{p \mid n} \eta\left(p^{r} \mid D(p)\right)
$$

Remark 4.10. Let $\{\chi\}$ be the set of Dirichlet's characters modulo $n$ and $\varphi(n):=\# \mathbb{Z}_{n}^{*}$. Then $W(\alpha ; n)$ is written by

$$
W(\alpha ; n):=\frac{1}{\varphi(n)} \sum_{\chi \bmod n} \chi\left(\alpha^{-1}\right) \xi(n) \sum_{m \geq 1, \operatorname{gcd}(n, m)=1} \chi(m) \beta(m) .
$$

Since $\chi\left(n_{1} n_{2}\right)=\chi\left(n_{1}\right) \chi\left(n_{2}\right)$ and $\beta\left(n_{1} n_{2}\right)=\beta\left(n_{1}\right) \beta\left(n_{2}\right)$ for relatively prime $n_{1}$, $n_{2}$, we get

$$
\sum_{m \geq 1, \operatorname{gcd}(n, m)=1} \chi(m) \beta(m)=\prod_{p \nmid n} \sum_{l \geq 0} \chi\left(p^{l}\right) \beta\left(p^{l}\right) .
$$

Calculating the above carefully, we can obtain the following expression of $W(\alpha ; n)$ like the Euler product.

$$
\begin{aligned}
W(\alpha ; n)= & \frac{1}{\varphi(n)} \sum_{\chi \bmod n} \chi\left(\alpha^{-1}\right) \prod_{p \geq 3, p \nmid n}\left(1+\frac{2 p^{2}(\chi(p)-1)}{\left(p^{2}-1\right)\left(p^{3}-\chi(p)\right)}\right) \\
& \times \begin{cases}1, & (2 \mid n), \\
\left(1+\frac{(\chi(2)-1)\left(32+4 \chi(2)+\chi(2)^{2}\right)}{24(8-\chi(2))}\right), & (2 \nmid n) .\end{cases}
\end{aligned}
$$

Proof. Let $T>0$ be a number with $T \ll x$. Then, according to Lemma 4.7, we have

$$
\pi(x ; D \equiv \delta \bmod n)=\pi(x ; D \equiv \delta \bmod n, u<T)+O\left(x^{2} T^{-2+\epsilon}\right) .
$$

Since $D(n)=u_{n}^{2} D$ for given $n$, the first term in the right hand side is described as follows.

$$
\begin{aligned}
\pi(x ; D \equiv \delta \bmod n, u<T) & =\sum_{m<T} \pi(x ; D \equiv \delta \bmod n, u=m) \\
& =\sum_{\alpha \in \mathbb{Z}_{n}^{*}} \sum_{\substack{m_{n} \equiv \alpha \bmod n \\
m<T}} \pi\left(x ; D(n) \equiv \delta \alpha^{2} \bmod n, u=m\right) .
\end{aligned}
$$


Here, the condition " $u=m$ " is equivalent to " $m \mid u$ and $m m_{1} \nmid u$ for any $m_{1}>1$ ". Then we have

$$
\begin{aligned}
& \pi(x ; D \equiv \delta \bmod n, u<T) \\
= & \sum_{\alpha \in \mathbb{Z}_{n}^{*}} \sum_{\substack{m_{n} \equiv \alpha \bmod \\
m<T}} \sum_{\substack{m \\
m_{1}<T / m}} \mu\left(m_{1}\right) \pi\left(x ; D(n) \equiv \delta \alpha^{2} \bmod n, m m_{1} \mid u\right) \\
= & \sum_{\alpha \in \mathbb{Z}_{n}^{*}} \sum_{\substack{m_{n} \equiv \alpha \bmod \\
m<T}} \sum_{\substack{m_{1}<T / m\\
}} \mu\left(m_{1}\right)\left(\eta\left(D(n) \equiv \delta \alpha^{2} \bmod n, m m_{1} \mid u\right) \operatorname{li}\left(x^{2}\right)\right. \\
& \left.+R\left(x ; D_{m} \equiv \delta \alpha^{2} \bmod n, m m_{1} \mid u\right)\right) \\
= & : S_{1}(T) \operatorname{li}\left(x^{2}\right)+S_{2}(T, x),
\end{aligned}
$$

where $\mu(m)$ is the Möbius function. Due to Lemma 4.1, 4.2 and 4.4, we get

$$
\begin{aligned}
& \left|S_{2}(U, x)\right| \\
\leq & C \sum_{\alpha \in \mathbb{Z}_{n}^{*}} \sum_{\substack{m_{n} \equiv \alpha \bmod \\
m<T}} \sum_{\substack{m<1 \\
m_{1}<T / m}} \eta\left(D_{m} \equiv \delta \alpha^{2} \bmod n, m m_{1} \mid u\right) v\left(n m_{1}\right) x^{3 / 2} \\
\leq & C \sum_{\alpha \in \mathbb{Z}_{n}^{*}} \sum_{\substack{m_{n} \equiv \alpha \bmod \\
m<T}} \sum_{n} n^{3} x^{3 / 2} \leq C^{\prime} n^{3} x^{3 / 2} T \log T
\end{aligned}
$$

for some $C>0$. On the other hand, since $\eta\left(D(n) \equiv \delta \alpha^{2} \bmod n, m m_{1} \mid u\right) \leq \eta\left(m m_{1} \mid u\right)=$ $v\left(m m_{1}\right)^{-1} \leq C\left(m m_{1}\right)^{-3}$, we have

$$
S_{1}(T)=\lim _{T \rightarrow \infty} S_{1}(T)+O\left(T^{-2}\right)
$$

Thus, combining (4.5), (4.6) and (4.7), we get

$$
\begin{aligned}
& \eta(D \equiv \delta \bmod n) \\
& =\sum_{\alpha \in \mathbb{Z}_{n}^{*}} \sum_{\substack{m_{n} \equiv \alpha \bmod \\
m<T}} \sum_{\substack{m_{1}<T / m \\
\text { m }}} \mu\left(m_{1}\right) \eta\left(D(n) \equiv \delta \alpha^{2} \bmod n, m m_{1} \mid u\right), \\
& R(x ; D \equiv \delta \bmod n)=O\left(x^{3 / 2} T \log T\right)+O\left(T^{-2+\epsilon} x^{2}\right) .
\end{aligned}
$$

Taking $T=x^{1 / 6}$, we can estimate the reminder term as in the theorem.

We now study $\eta(D \equiv \delta \bmod n)$. Denote by $n=p_{1}^{r_{1}} \cdots p_{l}^{r_{l}}$ the factorization of $n$. First calculate the sum over $m_{1}$.

$$
\begin{aligned}
& \sum_{m_{1}} \mu\left(m_{1}\right) \eta\left(D(n) \equiv \delta \alpha^{2} \bmod n, m m_{1} \mid u\right) \\
= & \sum_{m_{1}: \operatorname{gcd}\left(m_{1}, n\right)=1} \mu\left(m_{1}\right) \sum_{0 \leq k_{1}^{\prime}, \cdots, k_{l}^{\prime} \leq 1}(-1)^{k_{1}^{\prime}+\cdots+k_{l}^{\prime}} \eta\left(D(n) \equiv \delta \alpha^{2} \bmod n, p_{1}^{k_{1}^{\prime}} \cdots p_{l}^{k_{l}^{\prime}} m m_{1} \mid u\right) \\
= & \sum_{m_{1}: \operatorname{gcd}\left(m_{1}, n\right)=1} \mu\left(m_{1}\right) \eta\left(D(n) \equiv \delta \alpha^{2} \bmod n, m m_{1} \mid u, p_{i} m m_{1} \nmid u(\forall i)\right) .
\end{aligned}
$$


Next, take the sum over $m$. Due to Lemma 4.4, we have

$$
\begin{aligned}
& \sum_{\substack{m: m_{n} \equiv \alpha \bmod \\
n}} \sum_{\substack{m: \operatorname{gcd}(m, n)=1 \\
m \equiv \alpha \bmod n}} \sum_{\substack{m_{1}, \cdots, k_{l} \geq 0 \\
m_{1}: \operatorname{gcd}\left(m_{1}, n\right)=1}} \mu\left(m_{1}\right) \eta\left(D(n) \equiv \delta \alpha^{2} \bmod n, m m_{1} \mid u, p_{i} m_{1} \nmid u(\forall i)\right) \\
& \times \eta\left(D(n) \equiv \delta m^{2} \bmod n, m m_{1} \mid u, p_{i}^{k_{i}} \| u(\forall i)\right) \\
= & \sum_{\substack{m: \operatorname{gcd}(m, n)=1 \\
m \equiv \alpha \bmod n}} \mu\left(m_{1}\right) \eta\left(m m_{1} \mid u\right) \prod_{i=1}^{l}\left(\sum_{m_{i} \geq 0} \eta\left(D\left(p_{i}\right) \equiv n_{p_{i}}^{2} \delta \alpha^{2} \bmod p_{i}^{r_{i}}, p_{i}^{k_{i}} \| u\right)\right) \\
= & \prod_{i=1}^{l} \eta\left(D\left(m_{i}\right) \equiv n_{p_{i}}^{2} \delta \alpha^{2} \bmod p_{i}^{\left.r_{i}\right)} \sum_{\substack{m: \operatorname{gcd}(m, n)=1 \\
m \equiv \alpha \bmod n}} \sum_{m_{1}: \operatorname{gcd}\left(m_{1}, n\right)=1} \mu\left(m_{1}\right) \eta\left(m m_{1} \mid u\right) .\right.
\end{aligned}
$$

Let $m=q_{1}^{i_{1}} \cdots q_{t}^{i_{t}}$ be the factorization of $m$. Since $\eta(a b \mid u)=\eta(a \mid u) \eta(b \mid u)$ for relatively prime $a, b$, we have

$$
\begin{aligned}
& \sum_{m_{1}: \operatorname{gcd}\left(m_{1}, n\right)=1} \mu\left(m_{1}\right) \eta\left(m m_{1} \mid u\right) \\
= & \sum_{m_{1}: \operatorname{gcd}\left(m_{1}, n m\right)=1} \sum_{0 \leq j_{1}, \cdots, j_{t} \leq 1} \mu\left(q_{1}^{j_{1}} \cdots q_{t}^{j_{t}} m_{1}\right) \eta\left(q_{1}^{i_{1}+j_{1}} \cdots q_{t}^{i_{t}+j_{t}} m_{1} \mid u\right) \\
= & \sum_{m_{1}: \operatorname{gcd}\left(m_{1}, n m\right)=1} \mu\left(m_{1}\right) \eta\left(m_{1} \mid u\right) \prod_{k=1}^{t}\left(\eta\left(q_{k}^{i_{k}} \mid u\right)-\eta\left(q_{k}^{i_{k}+1} \mid u\right)\right) \\
= & \prod_{p \nmid n m}(1-\eta(p \mid u)) \prod_{k=1}^{t}\left(\eta\left(q_{k}^{i_{k}} \mid u\right)-\eta\left(q_{k}^{i_{k}+1} \mid u\right)\right) .
\end{aligned}
$$

Since $\eta(n \mid u)=v(n)^{-1}$, the above coincides $\xi(n) \beta(m)$ and then the expression of $\eta(D \equiv$ $\delta \bmod n)$ in the theorem follows immediately.

Example. As an example, we study the case of $n=5$. According to Theorem 4.8, we have

$$
\begin{aligned}
& \eta(D \equiv 0)=\eta(D(5) \equiv 0), \\
& \eta(D \equiv 1)=\eta(D(5) \equiv 1) W_{1}+\eta(D(5) \equiv 4) W_{2}, \\
& \eta(D \equiv 2)=\eta(D(5) \equiv 2) W_{1}+\eta(D(5) \equiv 3) W_{2}, \\
& \eta(D \equiv 3)=\eta(D(5) \equiv 3) W_{1}+\eta(D(5) \equiv 2) W_{2}, \\
& \eta(D \equiv 4)=\eta(D(5) \equiv 4) W_{1}+\eta(D(5) \equiv 1) W_{2},
\end{aligned}
$$

where $W_{1}:=W(1 ; 5)+W(4 ; 5)$ and $W(2):=W(2 ; 5)+W(3 ; 5)$. Due to Corollary 4.6, we see that

$$
\begin{array}{rlrl}
\eta(D(5) \equiv 0)=\frac{25}{62}, & \eta(D(5) \equiv 1)=\frac{63}{248}, & & \eta(D(5) \equiv 2)=\frac{125}{372} \\
\eta(D(5) \equiv 3)=\frac{1}{372}, & \eta(D(5) \equiv 4)=\frac{1}{248}
\end{array}
$$


We can compute that $W_{1}=0.80233 \cdots$ and $W_{2}=0.19766 \cdots$. Thus we get the following approximations.

$$
\begin{array}{lll}
\eta(D \equiv 0)=0.40322 \cdots, & & \\
\eta(D \equiv 1)=0.20461 \cdots, & & \eta(D \equiv 2)=0.27013 \cdots \\
\eta(D \equiv 3)=0.06857 \cdots, & & \eta(D \equiv 4)=0.05344 \cdots
\end{array}
$$

\subsection{For fundamental discriminants}

Let $d$ be $D$ when the square free factor of $D$ is 1 modulo 4 and $D / 4$ otherwise. Denote by $N_{\text {sf }}$ the set of positive square free integers. Raulf [Ra] proved that

$$
\begin{aligned}
\eta\left(d \in N_{\mathrm{sf}}\right) & =\frac{75}{112} \prod_{p \geq 3}\left(1-\frac{2 p}{p^{3}-1}\right)=0.42699 \cdots, \\
R\left(x ; d \in N_{\mathrm{sf}}\right) & =O\left(x^{2-\epsilon}\right)
\end{aligned}
$$

for some $\epsilon>0$. We now extend it as follows.

Theorem 4.11. Let $n \geq 1$ be an integer factored by $n=2^{e} \prod_{p \mid n} p^{r}$ and $\delta \in \mathbb{Z}_{n}$. Then $\eta\left(d \in N_{\mathrm{sf}}, d \equiv \delta \bmod n\right)$ exists and satisfies that

$$
\begin{aligned}
& \eta\left(d \in N_{\mathrm{sf}}, d \equiv \delta \bmod n\right) \\
= & \prod_{p \nmid 2 n}\left(1-\frac{2 p}{p^{3}-1}\right) \sum_{\alpha \in \mathbb{Z}_{n^{\prime}}^{*}} W\left(\alpha ; n^{\prime}\right) \\
& \times\left[\eta\left(D(2) \equiv 1 \bmod 4, D(2) \equiv \delta \alpha^{2} \bmod 2^{e}\right) \prod_{p \mid n} \eta\left(D(p) \equiv \delta \alpha^{2} \bmod p^{r}, p^{2} \nmid D\right)\right. \\
& \left.+\eta\left(D(2) \equiv 8,12 \bmod 16, D(2) \equiv 4 \delta \alpha^{2} \bmod 2^{e+2}\right) \prod_{p \mid n} \eta\left(D(p) \equiv 4 \delta \alpha^{2} \bmod p^{r}, p^{2} \nmid D\right)\right],
\end{aligned}
$$

where $n^{\prime}:=\operatorname{lcm}\left(16,4 n, \prod_{p \mid n} p^{2}\right)$. The reminder term is estimated by

$$
R\left(d \in N_{\mathrm{sf}}, d \equiv \delta \bmod n\right)=O\left(x^{25 / 13+\epsilon}\right) .
$$

Proof. Due to Lemma 4.7, we have

$$
\begin{aligned}
& \pi\left(x ; d \in N_{\mathrm{sf}}, d \equiv \delta \bmod n\right) \\
& =\pi\left(x ; d \in N_{\mathrm{sf}}, d \equiv \delta \bmod n, u<T\right)+O\left(T^{-2+\epsilon} x^{2}\right) .
\end{aligned}
$$

By the definition of $d$, we see that

$$
\begin{aligned}
& \pi\left(x ; d \in N_{\mathrm{sf}}, d \equiv \delta \bmod n, u<T\right) \\
= & \sum_{\substack{1 \leq m \leq x \\
\text { odd }}} \mu(m)\left(\pi\left(x ; D \equiv \delta \bmod n, D \equiv 1 \bmod 4, m^{2} \mid D, u<T\right)\right. \\
& \left.+\pi\left(x ; D \equiv 4 \delta \bmod 4 n, D \equiv 8,12 \bmod 16, m^{2} \mid D, u<T\right)\right) .
\end{aligned}
$$


Divide the sum above by

$$
\sum_{m<x}=\sum_{m<U}+\sum_{m \geq U}=: M_{1}(x, T, U)+M_{2}(x, T, U),
$$

where $U>0$ is a large number with $U \ll x$. According to Lemma 4.7, we have

$$
M_{2}(x, T, U)=O\left(U^{-1+\epsilon} x^{2}\right) .
$$

We further divide $M_{1}(x, T, U)$ by

$$
\begin{aligned}
M_{1}(x, T, U) & =\sum \pi(x ; \mathcal{C})=\sum \eta(\mathcal{C}) \operatorname{li}\left(x^{2}\right)+\sum R(x ; \mathcal{C}) \\
& =: M_{11}(T, U) \operatorname{li}\left(x^{2}\right)+M_{12}(x ; T, U)
\end{aligned}
$$

According to Corollary 4.6 and Theorem 4.8, we see that

$$
\eta\left(D \equiv \delta \bmod n, D \equiv 1 \bmod 4, m^{2} \mid D, u<U\right) \leq \eta\left(m^{2} \mid D\right) \leq C m^{-2}
$$

for some $C>0$ and $\eta\left(D \equiv 4 \delta \bmod 4 n, D \equiv 8,12 \bmod 16, m^{2} \mid D, u<U\right)$ is similarly. Then we have

$$
M_{11}(T, U)=\lim _{T, U \rightarrow \infty} M_{11}(T, U, x)+O\left(U^{-1}\right)
$$

On the other hand, by taking discussions similar to $S_{2}(T, x)$ in the proof of Theorem 4.8, we can estimate

$$
\left|M_{2}(T, U, x)\right| \leq C \sum_{m<U} n^{3} m^{4} x^{3 / 2} T \log T=O\left(U^{5} T^{1+\epsilon} x^{3 / 2}\right)
$$

Combining (4.8), (4.9), (4.11) and (4.12), we get

$$
\begin{aligned}
& \eta\left(d \in N_{\mathrm{sf}}, d \equiv \delta \bmod n\right) \\
& =\sum_{\substack{m \geq 1 \\
\text { odd }}} \mu(m)\left(\eta\left(D \equiv \delta \bmod n, D \equiv 1 \bmod 4, m^{2} \mid D\right)\right. \\
& \left.+\eta\left(D \equiv 4 \delta \bmod 4 n, D \equiv 8,12 \bmod 16, m^{2} \mid D\right)\right), \\
& R\left(x ; d \in N_{\mathrm{sf}}, d \equiv \delta \bmod n\right)=O\left(U^{-1+\epsilon} x^{2}\right)+O\left(T^{-2+\epsilon} x^{2}\right)+O\left(U^{5} T^{1+\epsilon} x^{3 / 2}\right) .
\end{aligned}
$$

Thus the estimate of the reminder term in the theorem follows immediately from the above with $T=x^{1 / 26}$ and $U=x^{1 / 13}$. 
We now arrange the expressions of $\eta\left(d \in N_{\text {sf }}, d \equiv \delta \bmod n\right)$. Change the sum over $m$ by

$$
\begin{aligned}
& \eta\left(d \in N_{\mathrm{sf}}, d \equiv \delta \bmod n\right) \\
= & \sum_{m: \operatorname{gcd}(m, 2 n)=1} \mu(m)(-1)^{j_{1}+\cdots+j_{l}} \\
& \times\left(\eta\left(D \equiv \delta \bmod n, D \equiv 1 \bmod 4, m^{2}\left|D, p_{i}^{2 j_{i}}\right| D(\forall i)\right)\right. \\
& \left.+\eta\left(D \equiv 4 \delta \bmod 4 n, D \equiv 8,12 \bmod 16, m^{2}\left|D, p_{i}^{2 j_{i}}\right| D(\forall i)\right)\right) \\
= & \quad \sum_{m: \operatorname{gcd}(m, 2 n)=1} \mu(m)\left(\eta\left(D \equiv \delta \bmod n, D \equiv 1 \bmod 4, m^{2} \mid D, p_{i}^{2} \nmid D(\forall i)\right)\right. \\
& \left.+\eta\left(D \equiv 4 \delta \bmod 4 n, D \equiv 8,12 \bmod 16, m^{2} \mid D, p_{i}^{2} \nmid D(\forall i)\right)\right) .
\end{aligned}
$$

According to Theorem 4.8, we have

$$
\begin{aligned}
& \eta\left(D \equiv \delta \bmod n, D \equiv 1 \bmod 4, m^{2} \mid D, p_{i}^{2} \nmid D(\forall i)\right) \\
= & \sum_{\alpha \in \mathbb{Z}_{n^{\prime} m^{2}}} W\left(\alpha ; n^{\prime} m^{2}\right) \eta\left(m^{2} \mid D\right) \eta\left(D(2) \equiv 1 \bmod 4, D(2) \equiv \delta \alpha^{2} \bmod 2^{e}\right) \\
& \times \prod_{i=1}^{l} \eta\left(D\left(p_{i}\right) \equiv \delta \alpha^{2} \bmod p_{i}^{r_{i}}, p_{i}^{2} \nmid D\right) .
\end{aligned}
$$

Similarly, we have

$$
\begin{aligned}
& \eta\left(D \equiv 4 \delta \bmod 4 n, D \equiv 8,12 \bmod 16, m^{2} \mid D, p_{i}^{2} \nmid D(\forall i)\right) \\
= & \sum_{\alpha \in \mathbb{Z}_{n^{\prime} m^{2}}^{*}} W\left(\alpha ; n^{\prime} m^{2}\right) \eta\left(m^{2} \mid D\right) \eta\left(D(2) \equiv 8,12 \bmod 16, D(2) \equiv 4 \delta \alpha^{2} \bmod 2^{e+2}\right) \\
& \times \prod_{i=1}^{l} \eta\left(D\left(p_{i}\right) \equiv 4 \delta \alpha^{2} \bmod p_{i}^{r_{i}}, p_{i}^{2} \nmid D\right) .
\end{aligned}
$$

Thus the following formula holds.

$$
\eta\left(d \in N_{\mathrm{sf}}, d \equiv \delta \bmod n\right)=\sum_{m \geq 1, \operatorname{gcd}(m, 2 n)=1} \mu(m) \eta\left(m^{2} \mid D\right) \sum_{\alpha \in \mathbb{Z}_{n^{\prime} m^{2}}} W\left(\alpha ; n^{\prime} m^{2}\right) \sigma\left(\alpha ; n^{\prime}\right),
$$

where

$$
\begin{aligned}
& \sigma\left(\alpha ; n^{\prime}\right) \\
:= & \eta\left(D(2) \equiv 1 \bmod 4, D(2) \equiv \delta \alpha^{2} \bmod 2^{e}\right) \prod_{p \mid n} \eta\left(D(p) \equiv \delta \alpha^{2} \bmod p^{r}, p^{2} \nmid D\right) \\
& +\eta\left(D(2) \equiv 8,12 \bmod 16, D(2) \equiv 4 \delta \alpha^{2} \bmod 2^{e+2}\right) \prod_{p \mid n} \eta\left(D(p) \equiv 4 \delta \alpha^{2} \bmod p^{r}, p^{2} \nmid D\right) .
\end{aligned}
$$


By the definition of $W(* ; *)$, we get

$$
\eta\left(d \in N_{\mathrm{sf}}, d \equiv \delta \bmod n\right)=\sum_{m \geq 1, \operatorname{gcd}(m, 2 n)=1} \mu(m) \eta\left(m^{2} \mid D\right) \sum_{\alpha \in \mathbb{Z}_{n^{\prime}}^{*}} W\left(\alpha ; n^{\prime}\right) \sigma\left(\alpha ; n^{\prime}\right) .
$$

According to Remark 4.9, we have

$$
\eta\left(m^{2} \mid D\right)=\prod_{p \mid m} \eta\left(p^{i} \mid D(p)\right)=\prod_{p \mid m} \frac{2 p^{3-i}}{p^{3}-1}
$$

for $2 \nmid m$ and $m=\prod_{p \mid m} p^{i}$. Thus the desired result follows immediately.

Example. Study the case of $n=5$. According to Theorem 4.11, we have

$$
\begin{aligned}
\eta\left(d \in N_{\text {sf }}, d \equiv 0 \bmod 5\right)= & \Omega \eta(5 \| D(5))\{\eta(D(2) \equiv 1 \bmod 4) \\
& +\eta(D(2) \equiv 8,12 \bmod 16)\}, \\
\eta\left(d \in N_{\text {sf }}, d \equiv 1 \bmod 5\right)= & \Omega\left[V_{1} \eta(D(5) \equiv 1 \bmod 5)+V_{2} \eta(D(5) \equiv 4 \bmod 5)\right], \\
\eta\left(d \in N_{\text {sf }}, d \equiv 2 \bmod 5\right)= & \Omega\left[V_{1} \eta(D(5) \equiv 2 \bmod 5)+V_{2} \eta(D(5) \equiv 3 \bmod 5)\right], \\
\eta\left(d \in N_{\text {sf }}, d \equiv 3 \bmod 5\right)= & \Omega\left[V_{1} \eta(D(5) \equiv 3 \bmod 5)+V_{2} \eta(D(5) \equiv 2 \bmod 5)\right], \\
\eta\left(d \in N_{\text {sf }}, d \equiv 4 \bmod 5\right)= & \Omega\left[V_{1} \eta(D(5) \equiv 4 \bmod 5)+V_{2} \eta(D(5) \equiv 1 \bmod 5)\right],
\end{aligned}
$$

where

$$
\begin{aligned}
& \Omega:=\prod_{p \neq 2,5}\left(1-\frac{2 p}{p^{3}-1}\right)=0.69357 \cdots \\
& V_{1}:=W_{1} \eta(D(2) \equiv 1 \bmod 4)+W_{2} \eta(D(2) \equiv 8,12 \bmod 16), \\
& V_{2}:=W_{1} \eta(D(2) \equiv 8,12 \bmod 16)+W_{2} \eta(D(2) \equiv 1 \bmod 4) .
\end{aligned}
$$

Since $W_{1}, W_{2}$ and $\eta(D(5) \equiv \delta \bmod 5)$ are given in the example for Theorem 4.8 and

$$
\begin{aligned}
& \eta(D(2) \equiv 1 \bmod 4)=\frac{19}{56}, \quad \eta(D(2) \equiv 8,12 \bmod 16)=\frac{37}{112} \\
& \eta(5 \| D(5))=\eta(5 \mid D(5))-\eta(25 \mid D(5))=\frac{10}{31}
\end{aligned}
$$

we get the following approximations.

$$
\begin{array}{ll}
\eta\left(d \in N_{\mathrm{sf}}, d \equiv 0\right)=0.1498 \cdots, & \\
\eta\left(d \in N_{\mathrm{sf}}, d \equiv 1\right)=0.0603 \cdots, & \eta\left(d \in N_{\mathrm{sf}}, d \equiv 2\right)=0.0792 \cdots \\
\eta\left(d \in N_{\mathrm{sf}}, d \equiv 3\right)=0.0780 \cdots, & \eta\left(d \in N_{\mathrm{sf}}, d \equiv 4\right)=0.0594 \cdots
\end{array}
$$

\section{References}

[Ar] E. Artin, Über die Zetafunktionen gewisser algebraischer Zahlkörper, Math. Ann. 89(1923), 147-156. 
[Bur] M. Burger, Small eigenvalues of Riemann surfaces and graphs, Math. Z. 205 (1990), 395-420.

[Bu] P. Buser, Geometry and spectra of compact Riemann surfaces, Progress in Math. 106, Birkhäuser, (1992).

[Da] B. A. Datskovsky, A mean-value theorem for class numbers of quadratic extensions, Number theory and related analysis, Contemp. Math. 143, 179-242.

[DH] A. Deitmar and W. Hoffmann, Asymptotics of class numbers, Invent. Math. 160 (2005), 647-675.

[G] C. F. Gauss, Disquisitiones arithmeticae, Fleischer, Leipzig, (1801).

[GH] D. Goldfeld and J. Hoffstein, Eisenstein series of $\frac{1}{2}$-integral weight and the mean value of real Dirichlet L-series, Invent. Math. 80 (1985), 185-208.

[HarWr] G.H. Hardy and E.M. Wright, An introduction to the theory of numbers, Fifth edition, Oxford University Press, 1979.

[HW] Y. Hashimoto and M. Wakayama, Splitting density for lifting about discrete groups, Tohoku Math. J. 59 (2007), p.527-545.

[He] D. Hejhal, The Selberg trace formula of PSL $(2, \mathbb{R})$ I, II, Lec. Notes in Math. 548, 1001 Springer, (1976, 1983).

[JK] J. Jorgenson and J. Kramer, On the error term of the prime geodesic theorem, Forum Math. 14 (2002), 901-913.

[Ki] H. H. Kim, Functionality for the exterior square of $\mathrm{GL}_{4}$ and the symmetric fourth of $\mathrm{GL}_{2}$ (with Appendix 1 by D. Ramakrishnan and Appendix 2 by Kim and P. Sarnak), J. Amer. Math. Soc. 16 (2003), 139-183.

[KS] H. H. Kim and F. Shahidi, Cuspidality of symmetric powers with applications, Duke Math. J. 112 (2002), 177-197.

[Ko] S. Koyama, Prime geodesic theorem for arithmetic compact surfaces, IMRN 1998, 383-388.

[Li] R. Lipschutz, Sitzungber, Akad. Berlin (1865), 174-185.

[LS] W. Luo and P. Sarnak, Quantum ergodicity of eigenfunctions on $\mathrm{PSL}_{2}(\mathbb{Z}) \backslash H^{2}$, Publ. Math. IHES, 81 (1995), 207-237.

[LRS] W. Luo, Z. Rudnick and P. Sarnak, On Selberg's eigenvalue conjecture, Geom. Funct. Anal. 5 (1995), 387-401.

[Me] F. Mertens, Ueber einige asymptotische Gesetze der Zahlentheorie, J. Reine Angew. Math. 77 (1874), 289-338. 
[Mo] R. A. Mollin, Quadratics, CRC Press Series on Discrete Mathematics and Its Applications, CRC Press (1995).

[Ra] N. Raulf, Asymptotics of class numbers for progressions and for fundamental discriminants, Forum Math. 21 (2009), 221-257.

[Sa1] P. Sarnak, Class numbers of indefinite binary quadratic forms, J. Number Theory, 15 (1982), 229-247.

[Sa2] P. Sarnak, The arithmetic and geometry of some hyperbolic three-manifolds, Acta Math. 151 (1983), 253-295.

[Sa3] P. Sarnak, Class numbers of indefinite binary quadratic forms II, J. Number Theory, 21 (1985), 333-346.

[Se] A. Selberg, Harmonic analysis and discontinuous groups in weakly symmetric Riemannian spaces with applications to Dirichlet series, J. Indian Math. Soc. (N.S.), 20 (1956), 47-87.

[Sh] T. Shintani, On zeta functions associated with the vector space of quadratic forms, J. Fac. Sci. Univ. Tokyo Sect. 1A Math. 22 (1975), 25-65.

[Si] C. L. Siegel, The average measure of quadratic forms with given determinant and signature, Ann. of Math. II, 45 (1944), 667-685.

[Su] T. Sunada, L-functions in geometry and some applications, Curvature and topology of Riemannian manifolds (Katata, 1985), 266-284, Lecture Notes in Math. 1201, Springer, Berlin, 1986.

[Ta] T. Takagi, Über eine theorie des relativ abelschen Zahlkörpers, Journal of the College of Science, Imperial University of Tokyo 41(1920), 1-133.

[Tc] N. Tchebotarev, Die Bestimmung der Dichtigkeit einer Menge von Primzahlen, welch zu einer gegebenen Substitutionsklasse gehoren, Math. Ann. 95(1926), 191228.

[Vi] I. M. Vinogradov, Improvement of the remainder term of some asymptotic formulas, Izvestiya Akad. Nauk SSSR. Ser. Mat. 13, (1949), 97-110

[Zo] P.G. Zograf, Fuchsian groups and small eigenvalues of the Laplacian operator, Soviet Math. Dokl. 26 (1984), 1618-1621.

HASHIMOTO, Yasufumi

Institute of Systems and Information Technologies/KYUSHU, 7F 2-1-22, Momochihama, Fukuoka 814-0001, JAPAN

e-mail:hasimoto@isit.or.jp 